\title{
Literatürler Eşliğinde Konjenital Böbrek Anomalisi Bulunan Hastalarda Taş Hastalığı Tedavisinin Değerlendirilmesi
}

Engin Kaya ${ }^{1}$, Giray Ergin², Turgay Ebiloğlư ${ }^{3}$, Yusuf Kibar ${ }^{1}$

${ }^{1}$ Gülhane Askeri Tıp Akademisi, Üroloji Anabilim Dall, Ankara

${ }^{2}$ Koru Hastanesi, Üroloji Kliniği, Ankara

${ }^{3}$ Etimesgut Asker Hastanesi, Üroloji Kliniği, Ankara

$\ddot{U}$ riner sistemdeki anotomik bozukluklara bağlı olarak taş görülme sıklığı da artmaktadır. Normal anatomiye sahip taş hastalarına tedavi yaklaşımı belirli iken, anomalili böbreklerdeki taş hastalığında standardize edilmiş bir tedavi yöntemi yoktur. Ekstrakorporeal şok dalga litotripsi (ESWL), üreterorenoskopi (URS), fleksible üreterorenoskopi (F-URS), perkütan nefrolitotomi (PNL), retrograd intrarenal cerrahi (RIRS), minimal invazif PNL (Mini-Perc), mikroperkütan nefrolitotomi (microperc), laparoskopik ya da robotik gibi yöntemlerin başarı şansı normale göre daha düşüktür.

Çocukluk çağı taş hastalığı dünya genelinde artan bir sağlık problemidir (1). Çocuklarda üriner sistem taşı insidansı yaklaşı \% $\%$-3 arasındadır (2). Bu riskin anormal anatomiye sahip böbreklerde oluşan staz sonucu idrarda meydana gelen kristallerin atılımındaki gecikme, enfeksiyon ve yine bu çocuklarda muhtemel var olduğu düşünülen metabolik anomali riskinin yüksekliği nedeni ile arttığı bildirilmektedir (3-5). Çocukluk yaş grubunda taşın spontan olarak düşme ihtimali erişkine göre daha zayıftır ve buna bağlı olarak cerrahi işlem ya da invaziv girişim ihtimali daha yüksektir (6). Ancak üriner sistem anomalilerinin taş oluşum riskini artırıp artırmadığı halen tartışmalıdır (2).

Günümüzde taş cerrahisi konusundaki gelişmelere bağlı olarak minimal invaziv tedavi seçeneklerinin kullanımı artmaktadır. Bu gelişmelere paralel olarak anatomik bozukluğun getirdiği cerrahi yük, tedavi seçeneklerin gelişimi ile azalmaktadır. Taşsızlık oranları giderek artarken, üriner enfeksiyon ve renal fonksiyon kayıp oranlarının düştüğü gözlenmektedir.

\section{Üreteropelvik Bileşke Obstruksiyonu (ÜPBO)}

ÜPBO ile \%16-30 oranlarında böbrek taşı birlikteliği gözlenebilmektedir (7). ÜPBO'na sekonder olarak idrar stazı ve enfeksiyon gelişmektedir. Bu durum kristallerin agregasyonuna, nükleasyonun gelişimine, kristallerin büyümesine ve sonunda taşın oluşumuna neden olmaktadır. ÜPBO'nun düzeltilmesi ile yeni taş oluşumunun azalması bu teoriyi destekler niteliktedir (8-11). Husmann ve ark.'larının yaptıkları çalışmada ÜPBO ile birlikte taşı bulunan çocuk hastalara piyeloplasti uygulanmış ve cerrahiye rağmen \%68 oranında taşın tekrarladığı saptanmıştır. Bu bulgulara göre anatomik bozukluğun düzeltilmesi sonrası idrar stazı ve enfeksiyonun oranının azaltılmasının, tek başına taş oluşumuna yol açan bir durum olmadığı, bu duruma ek olarak metabolik bozuklukların da etkili olabileceği sonucuna varılmıştır (4). Cerrahi tedavi sonrası bu hastalarda; sıvı alımının artırılıp, diyet ile ilgili düzenlemeler yapılması, taşın çıkarılması sonrası antibiyoterapinin 3 ay devamı (sitrüvit taşları için), taş analizine göre spesifik medikal tedavi başlanması ile taş hastalığının tekrarlama riski azaltılabilmektedir (12).

ÜPBO ve taş birlikteliğinde açık piyelolitotomi ve piyeloplasti \%90 oranında başarı sağlayan cerrahi yöntemlerdir $(13,14)$. Günümüzde minimal invaziv tedavi yöntemleri açık cerrahi tekniklerin yerini almaya başlamıştır. Perkütan nefrolitotomi (PNL) ve antegrad perkutan endopiyelotomi yönteminde iyi hasta seçimi ile \%64-85 oranında başarı oranı sağlanabilmektedir $(15,16)$. Berkman ve ark.'larının çalışmasında, toplam 146 hasta 2 gruba ayrılmıștır. Grup 1'de sadece ÜPBO bulunan 105 hasta, grup 2'de ÜPBO ile birlikte aynı tarafta böbrek taşı bulunan 41 hasta değerlendirilmiştir. Grup 1'deki hastalara yalnızca antegrad endopiyelotomi uygulandığında başarı oranı \%71 olarak saptanırken, grup 2'deki hastalara ek olarak PNL uygulandığında başarı oranının \%90'a yükseldiği bildirilmiştir (17). Antegrad endopiyelotomi'nin renal fonksiyonları kötü olan hastalarda, hidronefroz derecesi yüksek olan hastalarda ve çaprazlayan damar varlığında uygulanamaması, temel dezavantajları olup açık ve laparoskopik pyeloplastiye göre daha düşük başarı oranları ile karşımıza çıkmaktadır (18).

Laparoskopik piyeloplasti veya robotik piyeloplastiye ek olarak fleksible/rijit üreterorenoskopi ile yüksek oranda başarı oranları bildirilmektedir. Moon ve ark.'ları yaptıkları çalışmada başarı oranını \%96.2 olarak saptamışlardır (19). Laparoskopik piyeloplasti ile birlikte piyelolitotomi uygulanan 123 hastalık bir derlemede ise taşsızlık oranı \%91.3 (\%75100), obstrüksiyondan kurtulma oranı ise \%96.1 (\%90-100) olarak bildirilmiştir (8,20-24). Cerrahi öncesi taş sayısı ve yerinin doğru olarak saptanması, piyelitotomi hattının piyeloplasti öncesinde belirlenmesi, forseps ile taşın çıkarılması esnasında üretere dikkat edilmesi, işlem esnasında floroskopi ve 2 kamera kullanılması anahtar noktalardır (8). PNL ve beraberinde laparoskopik pyeloplasti operasyonunda, laparoskopik pyeloplasti ve beraberinde taşın fleksible veya rijid renoskop kullanılarak basket katater ile çıkarılmasına göre, intraoperatif floroskopinin ve litotriptörlerin kullanımının 
mümkün olması nedeni ile taşsızlık \%100 oranında daha yüksektir. Floroskopi ve litotriptörlerin intraoperatif kullanım imkanı nedeni ile bu vakalarda taşın sayısı, boyutu kısıtlayıcı değildir (24). Robotik piyeloplasti ile birlikte piyelolitotomi uygulanan 47 hastalık bir derlemede taşsızlık oranı \%92.3 (\%80.1-100), obstrüksiyondan kurtulma ise \%100 olarak bildirilmiştir (8,25-31).

\section{Atnalı Böbrek Anomalisi}

Atnalı böbrek, en sık füzyon anomalisi olup insidansı 1/400-666 canlı doğum olarak rapor edilmiştir (32-34). Erkeklerde görülme sıklığı kızlara oranla 2 kat fazladır. Füzyone böbrekler daha kaudalde yerleşir ve rotasyon defektine bağlı olarak tüm kaliksler ve renal pelvis anterior yerleşimlidir. Malrote füze olmuş böbrek nedeni ile üreter renal pelvis birleşimi normalden daha süperiorda ve lateralde gerçekleşir (yüksek insersiyon) (35). Atnalı böbrekte gözlenen en sık komplikasyon taştır. Böbrek taşı yaklaşık olarak \%21-60 oranında atnalı böbrekli olguya eşlik eder (32). At nalı böbreğin innervasyonu normal anatomiye sahip böbrekler ile aynı olduğu için semptomatolojisi benzerdir. Bunun yanı sıra anormal vasküler komşulukları ve anatomisi değişmiş yapıları, tedavi seçimi sırasında önem arz etmektedirler. ÜPBO varlığı tedavinin başarısını ciddi ölçüde etkileyebileceği için seçilen tedavi öncesinde tedavi alternatifleri mutlaka detaylı olarak değerlendirilmelidir. ESWL, F-URS, PNL, laparoskopik ve açık cerrahi yaklaşımlar tedavi seçenekleri içerisinde yer almaktadır.

ESWL, atnalı böbreklerde kullanılan minimal invaziv tedavi seçeneklerinden biridir. Fragmantasyon ve taşsızlık oranları sırası ile \%80 ve \%28-\%80 arasında olduğu bildirilmektedir (36-38). Bu çalışmalarda fazla sayıda seans ve 4 mm'den küçük fragmante taşların bırakılmasının başarı olarak değerlendirilmesi sebebiyle yüksek başarı oranları göze çarpmaktadır. Üriner staz varlığı, toplayıcı sistem dilatasyonu ve üreteropelvik bileşkenin daha yukarda yerleşmesi taş parçacıklarının atılmasını zorlaştıran önemli faktörlerdir. Taşın lokalizasyonu ve taş yükü atnalı böbreklerde taşsızlık oranlarını anlamlı düzeyde etkilemektedir. ESWL'nin başarısını etkileyen faktörlerden biri taş boyutudur. Sheir ve ark.'larının yaptığı çalışmada $15 \mathrm{~mm}$ 'den küçük taşlarda başarı oranı \%79 olarak bildirilirken, taş boyutu $15 \mathrm{~mm}$ 'den büyük taşlarda başarı oranı \%53 olarak rapor edilmektedir (39). Diğer bir çalışmada ise; 10-15 mm üstü taşlarda PNL ile taşsızlık oranının daha yüksek olacağı bildirilmiştir (40). Yine Ray ve ark.'larının yaptığı ve bu konudaki en geniş serilerden biri olan çalışmada, 41 atnalı böbrekli hastaya ESWL uygulanmıştır. Sonuç olarak tek seans ile başarının \%25 ve tam taş temizlik oranının \%9.1 olduğu bildirilmiştir. Genel başarı oranı \%63.6, genel taşsızlık oranı ise; \%39.1 olarak tespit edilmiştir. Bu hastaların \%73'ü tekrarlayan ESWL seansı, PNL, URS gibi ek tedavi modalitelerine ihtiyaç duymuşlardır (41). Böbrek taşlarının ultrasonografik olarak lokalizasyonu, bu hastalarda böbreklerin daha mediale yerleşimi nedeni ile çok efektif değildir. Floroskopik odaklama durumunda taş vertebraların spinöz çıkıntılarına veya kemik pelvise süperpoze olup net olarak odaklanamayabilir. Bu nedenlerle atnalı böbrekli hastalara ESWL uygulanması durumunda, hastaların flank veya supin pozisyonunda ESWL masasına yatırılması taşların süperpoze olmasını engelleyip, floroskopi ile daha net görüntü alınmasına ve böylece litotriptörün odak- lanmasına olanak sağlamaktadır.

F-URS, lazer litotripsi ve nitinol basket uygulaması, minimal invaziv tedavi yöntemi olarak kullanımı son zamanlarda giderek artan bir tedavi yöntemidir. Böbrek taşı ile birliktelik gösteren atnalı anomalisinde de; PNL ve ESWL'ye alternatif olarak tercih edilmeye başlanmıştır. Molimard ve ark'larının çalışmasında 17 atnalı böbrekli hastanın 15 (\%88.2)'inde taşsızlık sağlanırken, 1 hastada 48 . saatte persistan hematüri, 1 hastada 3 gün hospitalizasyon gerektiren piyelonefrit rapor edilmiştir. (42). Atış ve ark.'larının yaptı̆̆ı bir çalışmada, F-URS'nin başarı oranı \%70 olarak rapor edilmiştir. Geri kalan \%30'luk başarısız operasyonların \%71'inin alt pol taşı olduğu ve aynı zamanda $22 \mathrm{~mm}$ 'den büyük taşlar olduğu bildirilmiştir. Üç hastada postoperatif ateş gözlenirken, 2 hastada transfüzyon gerektirmeyen hematüri izlenmiştir (43).

\section{Pelvik Böbrek}

Embriyonel dönemde böbreklerin normal lokalizasyonuna ulaşamaması neticesinde ektopik yerleşim meydana gelir. $\mathrm{Bu}$ anomalinin en sık görülen şekli, pelvik yerleşim olup sıklıkla sol böbrekte izlenir. Yaklaşık insidansı 2200-3000 doğumda 1 olarak bildirilmiştir $(44,45)$. Pelvik böbrekler sıklıkla malrote ve yüksek insersiyona sahip üretere sahiptirler. Bu bozukluk böbrekte üriner staza ve dolayısıyla taş formasyonuna yol açan predispozan bir faktördür (46). Pelvik böbrekteki taşa yaklaşım, atnalı böbrekteki taşa yaklaşıma benzerdir.

ESWL'nin, pelvik böbrekte uygulama zorlukları bulunsa da; tedavi seçenekleri arasında yerini almaktadır. Kemik pelvise bağlı olarak ultrasonik yöntemle taşı odaklamak oldukça zordur $(47,48)$. Bu nedenle ESWL anterior yaklaşımla uygulanmalıdır. Komşu organ yaralanma riskini en aza indirmek için işlem öncesi BT ile komşu bağırsaklar ve böbreğin anatomisi belirlenebilir. Pelvik böbrekte ESWL ile taşsızlık oranlar1 \%25-92 arası bildirilmektedir $(20,49)$.

Anatomik pozisyona bağlı cerrahinin zorluğu daha bükülebilir cihazların gelişimi ile aşılmaya başlanmıştır. F-URS kullanımı pelvik böbrekli olgularda giderek artmaktadır. Weizer ve ark.'ları 4 vakalık F-URS deneyiminde başarı oranını \%75 olarak bildirirken, Binbay ve ark.'ları 17 vakalık tek seans F-URS başarısını \%70.8 olarak rapor etmişlerdir. Bozkurt ve ark'larının çalışmasında ise başarı oranı \%84.7 dir. $\mathrm{Bu}$ çalışmada başarısızlığın nedenleri ise lokalizasyona bağlı olarak taşa ulaşılamaması ya da fragmante parçaların düşmemesi olarak belirtilmektedir $(33,50,51)$.

PNL, pelvik böbrekte uygulaması zor ve komplikasyon oranı yüksek bir tedavi yöntemidir. Ultrasonografi ve laparoskopik görüş kullanılarak yapılan çalışmalarda sınırlı sayıda vaka bildirilmiştir. Desai ve ark.'ları çalışmalarında 16 vakanın tümünde başarı sağlarlarken, \%18 oranında barsak yaralanması, hematom ve postoperatif ileus gibi ciddi komplikasyonlar bildirilmişlerdir (52). Laparoskopik görüş altında PNL'de yüksek başarı oranı bildirilse de; cerrahi deneyim gereksinimi, hastanede uzun kalış süresi ve ciddi komplikasyon oranları önemli dezavantajlar olarak göze çarpmaktadır (53-55).

Günümüzde laparoskopik girişimlerin yaygınlaşması ve teknolojik gelişmeler ile birlikte; pelvik ektopik böbreğe direkt laparoskopik görüş altında giriş sağlanmakta ve böylece litotripsi veya litotomi işlemi gerçekleştirilmektedir. 
Literatürde bu yöntem ile ilgili olarak taşsızlık oranları yüksek, komplikasyon oranlarının düşük olduğu bildirilmektedir (44). Robotik cerrahinin günümüzde hızla yaygınlaşması ile literatürde ÜPBO'nun eşlik ettiği pelvik ektopik taşlı böbrek vakasında robotik pyelolitotomi ve beraberinde pyeloplasti operasyonu bildirilmiştir (56). Pelvik ektopik taşlı böbreklerde laparoskopik yaklaşım ile pyelolitotomi operasyonu, trokar içerisinden aynı zamanda fleksible sistoskop veya renoskopun kullanılabilme şansı nedeni ile giderek yaygınlaşmaktadır. Özellikle ÜPBO'da olan pelvik böbrek içerisindeki taşların tedavisinde laparoskopik/robotik yaklaşım önerilmektedir. Taşsızlık oranları bu yöntemde \%80-100 arası bildirilmektedir (20).

Pelvik böbrekte taş tedavisi özetle; ÜPBO ekarte edildiği, taşın 2 cm'den küçük olduğu vakalarda ESWL veya URS ilk tedavi seçenekleri olarak önerilirken, 2 cm'den büyük taşlarda ve ÜPBO'nun eşlik ettiği vakalarda PNL veya laparoskopik tedavinin ilk tedavi yaklaşımı olarak uygulanması önerilmektedir (57).

Microperc uygulamaları da; ektopik böbrekteki taşların tedavisinde yerini almaktadır. Ultrasonografi yardımlı microperc girişlerinin yaygınlaştığı gözlenirken, laparoskopi yardımlı microperc uygulamalarının kolaylığını gösteren olgu sunumları literatürde yerini almaya başlamıştır $(58,59)$.

\section{Çapraz Renal Ektopi}

Çapraz renal ektopi insidansı 1000 canlı doğumda 1 olarak bildirilmektedir (45). Literatürde geniş seriler olmasa da; çapraz renal ektopi vakalarında $2 \mathrm{~cm}$ 'e kadar taşlarda ESWL'nin başarı şansı yüksektir $(60,61)$. Gupta ve ark.'ları çalışmalarında 4 çapraz renal ektopili hastaya PNL uygulamış ve komplikasyon olmaksızın, \%100 oranında başarı bildirilmişlerdir.

Çapraz renal ektopili böbreklerde taş tedavisinde seçilecek cerrahi yaklaşıma karar vermek için BT ile hastanın renal anatomisinin iyi değerlendirilmesi, beraberinde ek anomalinin olup olmadığının tespit edilmesi önemlidir. Modi ve ark.'ları, çapraz renal ektopisi, taşı ve ÜPBO olan bir pediatrik vakaya yaptıkları transperitoneal laparoskopik pyelolitotomi ve pyeloplasti uygulamış ve tam taşsızlık rapor etmişlerdir (62). F-URS'nin kullanımının artması ile çapraz renal ektopili hastalarda minimal invaziv tedavi yöntemleri gelişecektir.

\section{Vezikoüreteral Reflü (VUR)}

VUR'u bulunan çocuklarda taş insidansı yaklaşık \%8-18 arasında bildirilmektedir. Fizyopatolojide reflüye bağlı staz, enfeksiyon ve metabolik anormallikler suçlanmaktadır $(63,64)$. VUR'lu çocuklarda hiperkalsiüri ve hiperürikozüri görülme sıklığının sağlıklı çocuklara göre daha yüksek oranda olduğu tespit edilmiştir (\%18.7-\%21.3) $(63,64)$. Bu hasta grubunda üriner sistem taş hastalığına yaklaşımın prensipleri, nonreflüksif hastalara benzerdir.

\section{Otozomal Dominant Polikistik Böbrek Hastalığı (ODPKBH)}

ODPKBH en sık rastlanan kalıtsal böbrek hastalığı olup insidansı 500-1000 canlı doğumda bir olarak bildirilmektedir (65). Bu hastaların yaklaşık \%20-28'ine üriner sistem taş has- talığı eşlik etmektedir (66-70). Büyümüş kistlere bağlı olarak idrar stazının artışı anatomik olarak taş oluşumuna katkıda bulunurken, idrar pH'sinın, idrar sitrat ve magnezyum düzeylerinin ODPKBH'lığı bulunan kişilerde düşük olması metabolik olarak taş sıklığını artıran faktörlerdir (67-69). BT bu hastalarda tedavi seçimi için önemlidir.

Taş pozisyonu ve lokalizasyonu ESWL kararı vermek için önemli kriterler olup, Deliveliotis ve ark.'ları çalışmalarında taşsızlık oranını \%25 olarak bildirilmişlerdir (71).

F-URS, özellikle $2 \mathrm{~cm}$ ve daha küçük boyuttaki taşlarda tercih edilebilir bir tedavi seçeneğidir. Yili ve ark.'larının yaptığı 13 olguluk seride taşsızlık oranının ilk fleksible URS de \%84.5, ikinci URS' de \%92.3 olduğunu bildirilmiştir (72).

PNL, $2 \mathrm{~cm}$ üzeri taşlarda ilk tercih edilen yöntemdir. Al-Kandari ve ark.'larının 20 olguluk, Srivastava ve ark.'larının 22 olguluk ve Lei ve ark'larının 23 olguluk çalışmalarında sırasıyla taşsızlık oranı \%89.4, \%88, \%95.7 olarak bildirilmiştir (73-75). PNL seçimindeki temel zorluk ilk girişte kist içine ve toplayıcı sisteme girilip girilemediğinin anlaşılamamasıdır. Toplayıcı sisteme girildiğini doğrulamak için retrograd olarak toplayıcı sistem metilen mavisi ile doldurulur. $\mathrm{Bu}$ sayede iğne ile giriş sonrası yapılan aspirasyonda metilen mavisinin geldiği görülerek toplayıcı sisteme giriş objektif olarak değerlendirilebilir.

Özetle, ODPKBH'nda 2 cm'den küçük taşlar için URS ve ESWL, 2 cm'den büyük taşlar için PNL tercih edilebilir.

\section{Kaynaklar}

1. VanDervoort K, Wiesen J, Frank R, et al. Urolithiasis in pediatric patients: a single center study of incidence, clinical presentation and outcome. J Urol. 2007;177:23002305.

2. Gupta, M. and Lee MW. Treatment of Stones associated with complex or anomalous renal anatomy. Urol Clin North Am 2007; 34: 431-41.

3. Rutchik, SD and Resnick MI. Ureteropelvic junction obstruction and renal calculi. Pathophysiology and implications for management. Urol Clin North Am 1998; 25: 317-21.

4. Husmann, DA, Milliner DS, and Segura JW. Ureteropelvic junction obstruction with concurrent renal pelvic calculi in the pediatric patient: a long-term followup. J Urol 1996; 156: 741-3.

5. Hsu, TH. and Streem SB. Metabolic abnormalities in patients with caliceal diverticular calculi. J Urol 1998; 160:1640-2

6. Pietrow PK, Pope JCt, Adams MC, Shyr Y, Brock JW III. Clinical outcome of pediatric stone disease. J Urol 2002;167(2 Pt 1):670-3

7. Pearle MS, Traxer O. Renal urolithiasis: therapy for special circumstances, part II, Lesson 40. AUA Update series, 2000; Lesson 40, 20:314

8. Skolarikos A, Dellis A, Knoll T. Ureteropelvic obstruction and renal stones: etiology and treatment. Urolithiasis. 2015; 43(1):5-12.

9. Burns JR, Finlayson B, Gauthier J (1984) Calcium oxalate retention in subjects with crystalluria. In: Ryall $\mathrm{R}$, Brockis JG, Marshall V (eds) Urinary stone: proceedings of the second international urinary stone conference. Churchill Livingstone, New York, pp 253-257 
10. Jenkins AD (1991) Calculus formation. In: Gillenwater JY, Grayhack JT, Howards SS et al (eds) Adult and pediatric urology, 2nd edn. Mosby Year Book, St Louis, pp 403-443

11. Bernardo NO, Liatsikos EN, Dinlenc CZ, Kapoor R, Fogarty JD, Smith AD. Stone recurrence after endopyelotomy. Urology 2000; 56: 378-381.

12. Hosking, D.H., S.B. Erickson, C.J. Van den Berg, et al. The stone clinic effect in patients with idiopathic calcium urolithiasis. J Urol 1983; 130: 1115-8

13. Arun N, Kekre NS, Nath V, et al. Is open pyeloplasty still justified? Br J Urol 1997; 80: 379-81.

14. O'Reilly PH, Brooman PJ, Mak S et al. The long-term results of Anderson-Hynes pyeloplasty. BJU Int 2001; 87: 287-9

15. Cassis, A.N., G.E. Brannen, W.H. Bush, et al. Endopyelotomy: review of results and complications. J Urol 1991; 146: 1492-5.

16. Motola JA, Badlani GH, Smith AD. Results of 212 consecutive endopyelotomies: an 8-year followup. J Urol 1993; 149: 453-6.

17. Berkman DS, Landman J, Gupta M. Treatment outcomes after endopyelotomy performed with or without simultaneous nephrolithotomy: 10-year experience. J Endourol 2009; 23:1409-1413

18. Knudsen BE, Cook AJ, Watterson JD, et al. Percutaneous antegrade endopyelotomy: long-term results from one institution. Urology 2004; 63: 230-4

19. Moon DA, El-Shazly MA, Chang CM et al. Laparoscopic pyeloplasty: evolution of a new gold standard. Urology 2006; 67: 932-6.

20. Ramakumar S, Lancini V, Chan DY, Parsons JK, Kavoussi LR, Jarrett TW. Laparoscopic pyeloplasty with concomitant pyelolithotomy. J Urol 2002; 167:1378-80.

21. Ball AJ, Leveillee RJ, Patel VR, Wong C. Laparoscopic pyeloplasty and flexible nephroscopy: simultaneous treatment of ureteropelvic junction obstruction and nephrolithiasis. JSLS 2004; 8:223-228

22. Srivastava A, Singh P, Gupta M, Ansari MS, Mandhani A, Kapoor R et al. Laparoscopic pyeloplasty with concomitant pyelolithotomy-is it an effective mode of treatment? Urol Int 2008; 80:306-309

23. Agarwal A, Varshney A, Bansal BS. Concomitant percutaneous nephrolithotomy and transperitoneal laparoscopic pyeloplasty for ureteropelvic junction obstruction complicated by stones. J Endourol 2008; 22: 2251-2255

24. Stein RJ, Turna B, Nguyen MM et al. Laparoscopic pyeloplasty with concomitant pyelolithotomy: technique and outcomes. J Endourol 2008; 22: 1251-1255

25. Atug F, Castle EP, Burgess SV, Thomas R. Concomtant management of renal calculi and pelvi-ureteric junction obstruction with robotic laparoscopic surgery. BJU Int 2005; 96:1365-1368

26. Palese MA, Stifelman MD, Munver R et al. Robot-assisted laparoscopic dismembered pyeloplasty: a combined experience. J Endourol 2005; 19:382-386.

27. Siddiq FM, Leveillee RJ, Villicana Pet al. Computerassisted laparoscopic pyeloplasty: University of Miami experience with the daVinci surgical system. J Endourol 2005; 19: 387-392

28. Mendez-Torres F, Woods M, Thomas R. Technical modifications for robot-assisted laparoscopic pyeloplasty. J Endourol 2005; 19:393-396
29. Mufarrij PW, Woods M, Shah OD, Palese MA, Berger AD, Thomas R, Stifelman MD. Robotic dismembered pyeloplasty: a 6-year, multi-institutional experience. J Urol 2008; 180: 1391-1396

30. Yanke BV, Lallas CD, Pagnani C, Bagley DH Robot assisted laparoscopic pyeloplasty: technical considerations and outcomes. J Endourol 2008; 22: 1291-1295

31. Nayyar R, Gupta N, Hemal A. Robotic management of complicated ureteropelvic junction obstruction. World J Urol 2010; 28 :599-602

32. Yohannes P, Smith AD. The endourological management of complications associated with horseshoe kidney. J Urol. 2002;168:5-8.

33. Weizer AZ, Silverstein AD, Auge BK, et al. Determining the incidence of horseshoe kidney from radiographic data at a single institution. J Urol. 2003;170:1722-1726.

34. Tepeler A, Sehgal PD, Akman T, Unsal A, Ozyuvali E, Armagan A, Nakada SY. Factors affecting outcomes of percutaneous nephrolithotomy in horseshoe kidneys. Urology. 2014; 84: 1290-4

35. Esuvaranathan, K., E.C. Tan, K.H. Tung, et al. Stones in horseshoe kidneys: results of treatment by extracorporeal shock wave lithotripsy and endourology. J Urol 1991; 146: 1213-5.

36. Chen WC., Lee YH, Huang JK, et al. Experience using extracorporeal shock-wave lithotripsy to treat urinary calculi in problem kidneys. Urol Int 1993; 51: 32-8.

37. Smith, J.E., K.N. Van Arsdalen, P.M. Hanno, et al. Extracorporeal shock wave lithotripsy treatment of calculi in horseshoe kidneys. J Urol 1989; 142: 683-6.

38. Kirkali Z, Esen AA, Mungan MU; Effectiveness of extracorporeal shockwave lithotripsy in the management of stone-bearing horseshoe kidneys. J Endourol 1996; 10: 13-5

39. Sheir, K.Z., K. Madbouly, E. Elsobky, et al. Extracorporeal shock wave lithotripsy in anomalous kidneys: 11-year experience with two second-generation lithotripters. Urology 2003; 62: 10-5; discussion 15-6.

40. Clayman RV. Effectiveness of extracorporeal shockwave lithotripsy in the management of stonebearing horseshoe kidneys. J Urol 1998; 160: 1949.

41. Ray AA, Ghiculete D, RJ DAH, et al. Shockwave lithotripsy in patients with horseshoe kidney: determinants of success. J Endourol 2011; 25: 487-93.

42. Molimard B, Al-Qahtani S, Lakmichi A, Sejiny M, GilDiez de Medina S, Carpentier X, Traxer O. Flexible ureterorenoscopy with holmium laser in horseshoe kidneys. Urology. 2010; 76: 1334-7

43. Atis, G., B. Resorlu, C. Gurbuz, et al. Retrograde intrarenal surgery in patients with horseshoe kidneys. Urolithiasis 2013; 41: 79-83.

44. Zafar, F.S. and J.E. Lingeman. Value of laparoscopy in the management of calculi complicating renal malformations. J Endourol 1996; 10: 379-83.

45. Shapiro E., Stuart B., Chow JS. Anomalies of the upper urinary tract. Campbell-Walsh Urology, 10th Edition. Editör: Wein AJ, Kavoussi L, Partin AW, Novick AC, Peters CA. Philadelphia USA, Elsevier Saunders, 2012; 3123-3160

46. Gleason, P.E., P.P. Kelalis, D.A. Husmann, et al. Hydronephrosis in renal ectopia: incidence, etiology and significance. J Urol 1994; 151: 1660-1. 


\section{LiTERATÜRLER EŞLIĞiNDE KONJENITAL BÖBREK ANOMALISi BULUNAN HASTALARDA TAŞ HASTALIĞI ...}

47. Baltacı S, Sarica K, Ozdiler E et al. Extracorporeal shockwave lithotripsy in anomalous kidneys. J Endourol 1994; 8: 179-81.

48. Talic RF. Extracorporeal shock-wave lithotripsy monotherapy in renal pelvic ectopia. Urology 1996; 48: 857-61.

49. Rigatti P, Montorsi F, Guazzoni G et al. Multimodal therapy for stones in pelvic kidneys. Urol Int 1991; 46: 29-34.

50. Binbay M, Skolarikos A, Unsal A, et al. Outcomes of retrograde intrarenal lithotripsy in pelvic kidneys. J Endourology. 2012; 20(Suppl):A154.

51. Bozkurt OF, Tepeler A, Sninsky B, Ozyuvali E, Ziypak T, Atis G, Daggulli M, Resorlu B, Caskurlu T, Unsal A. Flexible ureterorenoscopy for the treatment of kidney stone within pelvic ectopic kidney. Urology. 2014 84:12859

52. Desai MR, Jasani A. Percutaneous nephrolithotripsy in ectopic kidneys. J Endourol. 2000;14:289-292.

53. Matlaga BR, Kim SC, Watkins SL, et al. Percutaneous nephrolithotomy for ectopic kidneys: over, around, or through. Urology. 2006;67:513-517.

54. Gupta RC, Desai MR, Anil R, et al. Role of percutaneous nephrolithotomy in pelvic ectopic kidneys-17 years experience [abstract]. J Endourol. 2006;20(Suppl 1):A22.

55. Toth C, Holman E, Pasztor I, et al. Laparoscopically controlled and assisted percutaneous transperitoneal nephrolithotomy in a pelvic dystopic kidney. J Endourol. 1993;7:303-305.

56. Nayyar R, Singh P, Gupta NP. Robot-assisted laparoscopic pyeloplasty with stone removal in an ectopic pelvic kidney. JSLS. 2010; 14:130-2.

57. Tan, Y.K., D.Y. Cha, and M. Gupta. Management of stones in abnormal situations. Urol Clin North Am 2013; 40: 79-97.

58. Tepeler, A., M.S. Silay, A. Armagan, et al. Laparoscopicassisted "microperc" of a stone in a pelvic kidney of a 3-year-old girl. J Laparoendosc Adv Surg Tech A 2013; 23: 174-6.

59. Ganesamoni, R., R.B. Sabnis, S. Mishra, et al. Microperc for the management of renal calculi in pelvic ectopic kidneys. Indian J Urol 2013; 29: 257-9.

60. Romans DG, Jewett MA, Robson CJ. Crossed renal ectopia with colic. A clinical clue to embryogenesis $\mathrm{Br}$ J Urol 1976; 48:171-174

61. Semerci B, Verit A, Nazli O, Ilbey O, Ozyurt C, Cikili $\mathrm{N}$. The role of ESWL in the treatment of calculi with anomalous kidneys. Eur Urol 1997; 31:302-304

62. Modi P, Goel R, Dodia S. Case report: laparoscopic pyeloplasty with pyelolithotomy in crossed fused ectopia. J Endourol 2006; 20: 191-3
63. Rizvi SA, Naqvi SA, Hussain Z et al. Pediatric urolithiasis: developing nation perspectives. J Urol 2002; 168: 1522-5.

64. Noe HN, Stapleton FB, Jerkins GR et al. Clinical experience with pediatric urolithiasis. J Urol 1983; 129: 1166-8.

65. Mufti, U.B. and S.K. Nalagatla (2010) Nephrolithiasis in autosomal dominant polycystic kidney disease. J Endourol 24: 1557-61.

66. Ng CS, Yost A, Streem SB. Nephrolithiasis associated with autosomal dominant polycystic kidney disease: Contemporary urological management. J Urol. 2000; 163:726-729.

67. Torres VE, Wilson DM, Hattery RR, et al. Renal stone disease in autosomal dominant polycystic kidney disease. Am J Kidney Dis. 1993;22:513-519.

68. Torres VE, Erickson SB, Smith LH, et al. The association of nephrolithiasis and autosomal dominant polycystic kidney disease. Am J Kidney Dis. 1988;11:318-325.

69. Grampsas SA, Chandhoke PS, Fan J, et al. Anatomic and metabolic risk factors for nephrolithiasis in patients with autosomal dominant polycystic kidney disease. Am J Kidney Dis. 2000;36:53-57.

70. Dimitrakov D, Simeonov S. Studies on nephrolithiasis in patients with autosomal dominant polycystic kidney disease. Folia Med. 1994;436:27-30.

71. Deliveliotis, C., V. Argiropoulos, J. Varkarakis, et al. Extracorporeal shock wave lithotripsy produces a lower stone-free rate in patients with stones and renal cysts. Int J Urol 2002; 9: 11-4.

72. Yili L, Yongzhi L, Ning L et al. Flexible ureteroscopy and holmium laser lithotripsy for treatment of upper urinary tract calculi in patients with autosomal dominant polycystic kidney disease. Urol Res 2012; 40: 87-91

73. Al-Kandari, AM, Shoma AM, Eraky I et al. Percutaneous nephrolithotomy for management of upper urinary tract calculi in patients with autosomal dominant polycystic kidney disease. Urology 2009; 74: 273-7.

74. Srivastava A, Bansal R, Srivastava A et al. Percutaneous nephrolithotomy in polycystic kidney disease: is it safe and effective? Int Urol Nephrol 2012; 44: 725-30.

75. Lei M, Zhu W, Wan SP, Liu Y, Zeng G, Yuan J. Safety and efficacy of minimally invasive percutaneous nephrolithotomy in patients with autosomal dominant polycystic kidney disease. J Endourol 2014; 28: 17-22.

Yazışma Adresi:

Yusuf Kibar

GATA Askeri Tıp Fakültesi, Anabilim Dalı, Ankara

Tel: +90 5325626192

e-mail:ykibar@gata.edu.tr 